DIGITALCOMMONS - @WAYNESTATE -
Clinical Research in Practice: The Journal of Team Hippocrates

Volume 5 | Issue 2

Article 6

2019

\title{
Andexanet alfa is an effective reversal agent for factor Xa inhibitors in patients that develop intracranial hemorrhage
}

Joseph Friedli

Wayne State University School of Medicine, jfriedli@med.wayne.edu

Follow this and additional works at: https://digitalcommons.wayne.edu/crp

Part of the Chemical and Pharmacologic Phenomena Commons, Medical Education Commons, Other Chemicals and Drugs Commons, Pharmaceutical Preparations Commons, and the Translational Medical Research Commons

\section{Recommended Citation}

FRIEDLI J. Andexanet alfa is an effective reversal agent for factor Xa inhibitors in patients that develop intracranial hemorrhage. Clin. Res. Prac. 2019 Sep 6;5(2):eP1854. doi: 10.22237/crp/1567555500

This Critical Analysis is brought to you for free and open access by the Open Access Journals at DigitalCommons@WayneState. It has been accepted for inclusion in Clinical Research in Practice: The Journal of Team Hippocrates by an authorized editor of DigitalCommons@WayneState. 


\title{
Andexanet alfa is an effective reversal agent for factor $\mathrm{Xa}$ inhibitors in patients that develop intracranial hemorrhage
}

JOSEPH FRIEDLI, Wayne State University School of Medicine, jfriedli@med.wayne.edu

\begin{abstract}
A critical appraisal and clinical application of Connolly SJ, Milling TJ, Eikelboom JW, et al. Andexanet alfa for acute major bleeding associated with factor Xa inhibitors. N Engl J Med. 2016;375(12):1131-1141. doi: 10.1056/NEJMoa1607887.
\end{abstract}

Keywords: $\quad$ Factor Xa inhibitor reversal, intracranial hemorrhage

\begin{abstract}
Clinical Context
Clarence Williams [pseudonym], a 78-year-old African-American male, presented with mild chest pain and shortness of breath. He was found to have bilateral pulmonary emboli, diagnosed by CT pulmonary angiogram. One month prior, he underwent a bifrontal craniotomy and gross total excision of a pituitary macroadenoma with a long hospitalization. Past medical history was also significant for hypertension, coronary artery disease, central hypothyroidism, hypothalamic-pituitary-adrenal suppression, and type 2 diabetes mellitus. CT head was negative for an acute bleed and he was started on low intensity heparin. Medical decision-making then trended towards maintenance of anticoagulation. Mr. Williams was initially agreeable to using apixaban, a direct oral anticoagulant (DOAC), for treatment once his condition stabilized. Due to the inconveniences of regular blood checks requiring constant transportation, Mr. Williams preferred the more expensive apixaban over warfarin. Given his comorbidities and age, he stated a desire to limit the amount of medical appointments requiring travel. However, the neurosurgery team consulted for Mr. Williams' case recommended against apixaban due to the high risk for major bleeding. Per neurosurgery, the patient was started on enoxaparin with a bridge to warfarin due to the convenient reversibility of warfarin in the case of intracranial hemorrhage. The primary care team wondered about the efficacy of potential options for reversing direct oral anticoagulants, given that Mr. Williams' or iginal treatment preference was apixaban.
\end{abstract}

\section{Clinical Question}

What is the efficacy of reversing factor Xa inhibitors in the case of intracranial hemorrhage?

\section{Research Article}

Connolly SJ, Milling TJ, Eikelboom JW, et al. Andexanetalfa for acute major bleeding associated with factor Xa inhibitors. N Engl J Med. 2016;375(12):1131-1141. doi: 10.1056/NEJMoa1607887

JOSEPH FRIEDLI is a student at Wayne State University School of Medicine. 
FRIEDLI J. Andexanet alfa is an effective reversal agent for factor Xa inhibitors in patients that develop intracranial hemorrhage. Clin. Res. Prac. 2019 Sep 6;5(2):eP1854. doi: 10.22237/crp/1567555500
Clinical Research in Practice

The Journal of Team Hippocrates

VOL 5 ISS 2 / eP1854 / SEPTEMBER 6, 2019 doi: $10.22237 / \mathrm{crp} / 1567555500$

\section{Related Literature}

Utilizing PubMed Advanced Search Builder, title and abstract terms used as input included "factor Xa inhibitor" AND "major bleeding" NOT "atrial fibrillation". As of September 2018, the search returned 99 total citations; 42 of these were clinical trials and were reviewed for application to the question. The Clinical Queries and Similar Articles options on PubMed were also employed to ensure all pertinent articles were reviewed. Although there is significant research on direct oral anticoagulants, the ongoing trials to discover an efficacious option for apixaban reversal narrowed this search. UpToDate was used as an adjunct for informational purposes.

Tao et al. conducted a retrospective study that determined the safety of 4 -factor prothrombin complex concentrate (4F-PCC) as a reversal agent for patients taking factor Xa inhibitors. 1 The rate of thromboembolism in 43 such patients was $2.1 \%(1 / 43)$, with the authors judging the 4F-PCC to be acceptable as a viable reversal option. However, establishing the safety, rather than efficacy, of 4FPCC for anticoagulation reversal was the primary goal of this study, so this article did not fit the clinical question asked.

Di Nisio et al. explored the predictive risk factors of major bleeding in patients receiving rivaroxaban or heparin and vitamin $\mathrm{K}$ antagonists for treatment of venous thromboembolism. $\underline{2}$ This study analyzed data from phase III randomized, open-label trials. Older age, black race, low hemoglobin concentrations, cancer, and antiplatelet or non-steroidal anti-inflammatory drug therapy all contribute to a higher risk of major bleeding events. Because this study was not specifically targeting the reversal of facto $\mathrm{r}$ Xa inhibitors, it cannot answer the clinical question.

Gomez et al. performed a prospective, non-interventional, phase IV study of thromboembolic and bleeding events in patients being treated with rivaroxaban following total joint arthroplasty. $\underline{3}$ The study demonstrated that rivaroxaban was protective against pulmonary embolism, our patient's clinical problem. However, our patient underwent craniotomy and not arthroplasty, so our patient does not match this study's population.

The article by Connolly et al. is a prospective study of andexanet alfa in 67 patients receiving a factor Xa inhibitor who developed acute major bleeding. -4 This ongoing, multicenter investigation aims to address the efficacy and safety of reversing factor Xa inhibitors with andexanet alfa to achieve effective hemostasis. It is the most current study relevant to our patient's preferences and health risks and no other studies investigating the efficacy of andexanet alfa are published. Therefore, it is the best artic le to tackle the clinical question asked.

\section{Critical Appraisal}

The article by Connolly et al. is a prospective study of andexanet alfa in 67 patients receiving a factor Xa inhibitor (apixa ban, rivaroxaban, edoxaban, or enoxaparin) who developed acute major bleeding. The purpose for this ongoing investigation is to evaluate andexanet as an efficacious reversal agent for factor Xa inhibitors.

Connolly et al. performed a single-group cohort study instead of a randomized clinical trial due to logistical and ethical issues. There is no standard of care established for reversal of anticoagulation from factor Xa inhibitors and it would be unethical to use a pla cebo control in the case of uncontrolled major bleeding. Because this was a single-group cohort without a control, statistical analysis of risk reduction and number needed to treat are not calculable.

The 67 patients enrolled in the study had a mean age of 77 years and all of them had a thrombotic event and cardiovascular di sease history. Patients were only eligible if they were at least 18 years of age and underwent treatment with one of four factor Xa inhibitors. Enrollment was limited to those who developed severe bleeding who required urgent reversal of anticoagulation. Because the criteria are restrictive, it is difficult to examine a representative population sample, which diminishes the statistical power of the study.

This study analyzed the safety of andexanet in all 67 patients. Investigation of efficacy included only 47 of these subjects who met criteria for baseline anti-factor Xa activity determined through chromogenic assay, or acute major bleeding. An adjudication committee assessed the severity of bleeding and established the specific inclusion criteria. As this study is ongoing, the au thors 
FRIEDLI J. Andexanet alfa is an effective reversal agent for factor Xa inhibitors in patients that develop intracranial hemorrhage. Clin. Res. Prac. 2019 Sep 6;5(2):eP1854. doi: 10.22237/crp/1567555500
Clinical Research in Practice

The Journal of Team Hippocrates

VOL 5 ISS 2 / eP1854 / SEPTEMBER 6, 2019 doi: $\underline{10.22237 / \mathrm{crp} / 1567555500}$

should continue enrolling patients in order to achieve better statistical power for efficacy and safety analyses. The Level of Evidence is 2 according to the SORT criteria based on a cohort study. $\underline{5}$

Notably, this research is financially supported by Portola Phar maceuticals, the developer of andexanet alfa. Therefore, there is a potential funding bias in this study, but it is difficult to determine how much of an impact is present.

Regarding efficacy, the authors determined that 37 of their 47 patients (79\%) achieved excellent or good hemostasis in the 12 hours following andexanet administration. The terms excellent and good were both considered effective, versus poor or none which we re considered not effective. For intracranial bleeds, excellent and good were defined by increases in hematoma volume of $20 \%$ and $35 \%$ or less from baseline, respectively. Serial CT or MRI scans compared the changes in hematoma volume from baseline at both 1 hour and 12 hours following infusion. Of the 47 patients, 20 were those with intracranial bleeding, with an $80 \%$ achievement rate of excellent or good efficacy.

The patient population for efficacy in this study was similar to our patient in question. His age (78) and body-mass index (29) were near the means (77 and 28.8, respectively), his indication for anticoagulation was pulmonary embolism (9\% of patients), and his medical history included diabetes mellitus (36\% of patients). While our patient had other characteristics that did not match the majority of subjects in this study, such as white race and atrial fibrillation, he did not have any features that would have prevented him from enrolling in the trial had he been treated with a factor Xa inhibitor and developed an acute bleeding event. Therefore, the results of this study can be applied to our question.

\section{Clinical Application}

This study by Connolly et al. demonstrates that $79 \%$ of patients achieved excellent or good hemostasis following andexanet administration, due to rapid reversal of anti-Xa activity. Mr. Williams was initially amenable to using a factor Xa inhibitor for treatment of bilateral pulmonary emboli. His preference of the more expensive apixaban over the reversible warfarin was rooted in the inconvenience of regular blood draws to monitor anticoagulation. As an elderly gentleman with extensive medical comorbidities, constant travel to-and-from appointments was an impeding factor in his decision. Unfortunately, his preferences did not outweigh the neurosurgery team's recommendations.

Mr. Williams does fit the appropriate enrollment profile that is concordant with the subject population of this project. The advantage of applying this research to clinical practice is to establish a viable option for reversing factor Xa inhibitors in the case of acute major bleeding. Although our patient's medical management ultimately did not include a factor $X a$ inhibitor, this article addresses the clinical question. This study is still ongoing, but the preliminary results are promising and suggest that the status of andexanet alfa as an efficacious reversal agent is on the horizon.

Learning points:

1. Andexanet alfa is a modified human factor Xa decoy protein specifically designed to reverse factor Xa inhibitors.

2. For patients on factor Xa inhibitors who develop intracranial hemorrhage, andexanet alfa is a promising option for reversal.

3. Further research is warranted for establishing the efficacy of andexanet alfa as a reversal agent.

\section{References}

1. Tao J, Bukanova EN, Akhtar S. Safety of 4-factor prothrombin complex concentrate (4F-PCC) for emergent reversal of factor Xa inhibitors. Journal of Intensive Care. 2018;6:34. doi: 10.1186/s40560-018-0303-y. 
2. Di Nisio M, Ageno W, Rutjes AW, et al. Risk of major bleeding in patients with venous thromboembolism treated with rivaroxaban or with heparin and vitamin K antagonists. Thrombosis and Haemostasis. 2016;115(2):424-432. doi: 10.1160/th1506-0474.

3. Gomez D, Razmjou H, Donovan A, et al. A Phase IV Study of Thromboembolic and Bleeding Events Following Hip and Knee Arthroplasty Using Oral Factor Xa Inhibitor. The Journal of Arthroplasty. 2017;32(3):958-964. doi: 10.1016/i.arth.2016.09.021.

4. Connolly SJ, Milling TJ, Eikelboom JW, et al. Andexanet Alfa for Acute Major Bleeding Associated with Factor Xa Inhibitors. The New England Journal of Medicine. 2016;375(12):1131-1141. doi: 10.1056/NEJMoa1607887.

5. Ebell MH, Siwek J, Weiss BD, et al. Strength of Recommendation Taxonomy (SORT): A Patient-Centered Approach to Grading Evidence in the Medical Literature. American Family Physician. https://www.aafp.org/afp/2004/0201/p548.html. Published February 1, 2004. Accessed July 15, 2019. 